\title{
Increased Tpeak-Tend interval is highly and independently related to arrhythmic events in Brugada syndrome
}

Philippe Maury, MD, ${ }^{*}$ Frederic Sacher, MD, Jean-Baptiste Gourraud, MD, ${ }^{\ddagger}$ Jean-Luc Pasquié, MD, ${ }^{\S}$ Franck Raczka, MD, ${ }^{\S}$ Vanina Bongard, PhD, ${ }^{*}$ Alexandre Duparc, MD, Pierre Mondoly, $M D^{*}{ }^{*}$ Marie Sadron, $M D,{ }^{*}$ Stephanie Chatel, $\mathrm{PhD}^{\ddagger}{ }^{\ddagger}$ Nicolas Derval, $\mathrm{MD}^{\dagger}{ }^{\dagger}$ Arnaud Denis, MD, ${ }^{\dagger}$ Christelle Cardin, MD, Jean-Marc Davy, MD, ${ }^{\S}$ Meleze Hocini, MD, ${ }^{\dagger}$ Pierre Jaïs, MD, ${ }^{\dagger}$ Laurence Jesel, MD, ${ }^{\dagger}$ Didier Carrié, MD, ${ }^{*}$ Michel Galinier, MD, Michel Haïssaguerre, MD, ${ }^{\dagger}$ Vincent Probst, MD, ${ }^{\ddagger}$ Anne Rollin, $M D^{\star}$

From the *University Hospital Rangueil, Toulouse, France, ${ }^{\dagger}$ University Hospital Haut-Leveque, Bordeaux-Pessac, France, ${ }^{\ddagger}$ Institut du Thorax, Nantes, France, and ${ }^{\S}$ University Hospital Arnaud de Villeneuve, Montpellier, France.

BACKGROUND Risk stratification in Brugada syndrome (BS) remains controversial. The time interval between the peak and the end of the T wave (Tpe interval), a marker of transmural dispersion of repolarization, has been linked to malignant ventricular arrhythmias in various settings but leads to discordant results in BS.

OBJECTIVE We study the correlation of the Tpe interval with arrhythmic events in a large cohort of patients with BS.

METHODS A total of 325 consecutive patients with BS (mean age $47 \pm 13$ years, 259 men- $80 \%)$ with spontaneous $(n=143,44 \%)$ or drug-induced ( $\mathrm{n}=182,56 \%$ ) type 1 electrocardiogram were retrospectively included. 235 were asymptomatic $(70 \%), 80$ presented with unexplained syncope $(22 \%)$, and 10 presented with sudden death (SD) or appropriate implantable cardioverter-defibrillator therapy (AT) $(8 \%)$ at diagnosis or over a mean follow-up of $48 \pm 34$ months. The Tpe interval was calculated as the difference between the QT interval and the QT peak interval as measured in each of the precordial leads.

RESULTS The Tpe interval from lead $V_{1}$ to lead $V_{4}$, maximum value of the Tpe interval (max Tpe), and Tpe dispersion in all precordial leads were significantly higher in patients with SD/AT or in patients with syncope than in asymptomatic patients $(P<.001)$. A max Tpe of $\geq 100 \mathrm{~ms}$ was present in 47 of 226 asymptomatic patients (21\%), in
48 of 73 patients with syncope $(66 \%)$, and in 22 of 26 patients with SD/AT $(85 \%)(P<.0001)$. In multivariate analysis, a max Tpe of $\geq 100 \mathrm{~ms}$ was independently related to arrhythmic events (odds ratio 9.61; 95\% confidence interval 3.13-29.41; $P<.0001)$.

CONCLUSION The Tpe interval in the precordial leads is highly related to malignant ventricular arrhythmias in this large cohort of patients with BS. This simple electrocardiographic parameter could be used to refine risk stratification.

KEYWORDS Brugada syndrome; Transmural dispersion of repolarization; Sudden death; QT interval

ABBREVIATIONS AT = appropriate therapy; $\mathbf{B S}=$ Brugada syndrome; CI = confidence interval; $\mathbf{E C G}=$ electrocardiogram/ electrocardiographic; ICD = implantable cardioverter-defibrillator; Max Tpe = maximum value of the time interval between the peak and the end of the $\mathrm{T}$ wave; $\mathbf{O R}=$ odds ratio; $\mathbf{R O C}=$ receiver operating characteristic; RVOT = right ventricular outflow tract; SD $=$ sudden death; Tpe interval $=$ time interval between the peak and the end of the T wave

\section{Introduction}

Brugada syndrome (BS) is defined as $\geq 2 \mathrm{mV}$ coved ST segment elevation with negative $\mathrm{T}$ waves in the right precordial leads in patients without structural heart disease carrying the risk of sudden cardiac death (SD) due to malignant ventricular arrhythmias. ${ }^{1}$ To date, the implantable cardioverter-defibrillator (ICD) is the only validated therapy for patients with $\mathrm{BS}$, which are

Address reprint requests and correspondence: Dr Philippe Maury, Department of Cardiology, University Hospital Rangueil, 31059 Toulouse Cedex 09, France. E-mail address: mauryjphil@hotmail.com. proposed to symptomatic patients, while risk stratification in asymptomatic patients remains largely debated. The annual rate of cardiac events in asymptomatic patients with BS is however not negligible, ${ }^{2}$ and thus there is an urgent need for new reliable markers for SD in asymptomatic patients with BS.

The time interval between the peak and the end of the $T$ wave (Tpe interval) has been proposed as a marker of transmural dispersion of repolarization ${ }^{3}$ and has been shown to identify patients at higher risk of malignant arrhythmic events in various settings. ${ }^{4-10}$ A few studies ${ }^{11-14}$ have also addressed this issue in BS but have led to discordant results. 
The aim of this study was to investigate the feasibility of the evaluation of the Tpe interval, the correlation of the Tpe interval with the clinical or electrophysiological parameters and with the occurrence of arrhythmic events in a large cohort of patients with BS.

\section{Methods}

We performed a retrospective multicenter study, including consecutive patients with BS recruited at the French university hospitals of Nantes, Bordeaux, Toulouse, and Montpellier from 1996 to 2010. BS diagnosis was made according to the currently accepted criteria in the case of spontaneous or drug-induced type $1 \mathrm{ST}$ elevation in the right precordial leads. ${ }^{1}$ This study was approved by the institutional committee on human research at the authors' institution. Written informed consent was obtained for all the subjects who participated in the study.

For each patient, the 12-lead surface electrocardiogram (ECG) recorded at the time of diagnosis and showing the highest type 1 ST elevation was used for the analysis, either spontaneously or after provocative drug test (ajmaline $1 \mathrm{mg} / \mathrm{kg}$ over 5 minutes or flecainide $2 \mathrm{mg} / \mathrm{kg}$ over 10 minutes; paper speed $25 \mathrm{~mm} / \mathrm{s}$; amplification $1 \mathrm{~cm} / \mathrm{mV}$ ). The QT interval duration was measured between the QRS onset and the end of $\mathrm{T}$ wave, ${ }^{15}$ and the QT peak interval was measured between the QRS onset and the peak of the T wave. Leads with T-wave amplitude $<0.1 \mathrm{mV}$ or without discernible $\mathrm{T}$ wave (fully included in ST elevation) were not analyzed. The Tpe interval in each lead was then calculated by the difference between the QT interval and the QT peak interval (Figure 1A). Maximal Tpe interval (max Tpe) was defined as the maximum value of the Tpe interval in the precordial leads. We also calculated the Tpe/QT ratio and Tpe dispersion (defined as the difference between the maximum and the minimum value of the Tpe interval in the precordial leads). Peripheral ECG leads did not give any supplementary information through the intermediate analysis of the first 80 patients (data not shown), and therefore measurements were performed in the precordial leads only for the remaining patients.

ECG analysis and measurements were performed by a single observer blinded to the symptoms (A.R.). Measurements were independently performed by a second observer (P.M.) in a randomly selected subgroup of ECG (1 of 5 patients) in order to define the interobserver correlations.

Patients were divided into 3 groups depending on their symptoms and/or documented spontaneous ventricular arrhythmias: asymptomatic, unexplained syncope (supposed to be caused by malignant ventricular arrhythmia after exclusion of other causes such as neurocardiogenic syncope), and malignant ventricular arrhythmias: SD or

A
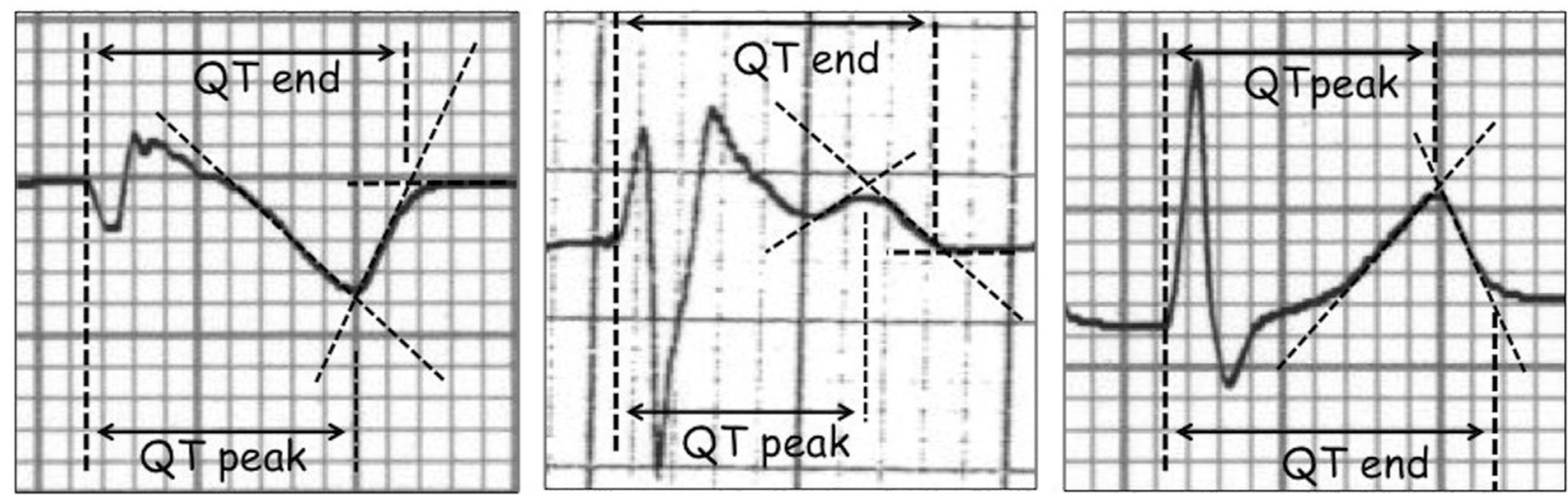

B
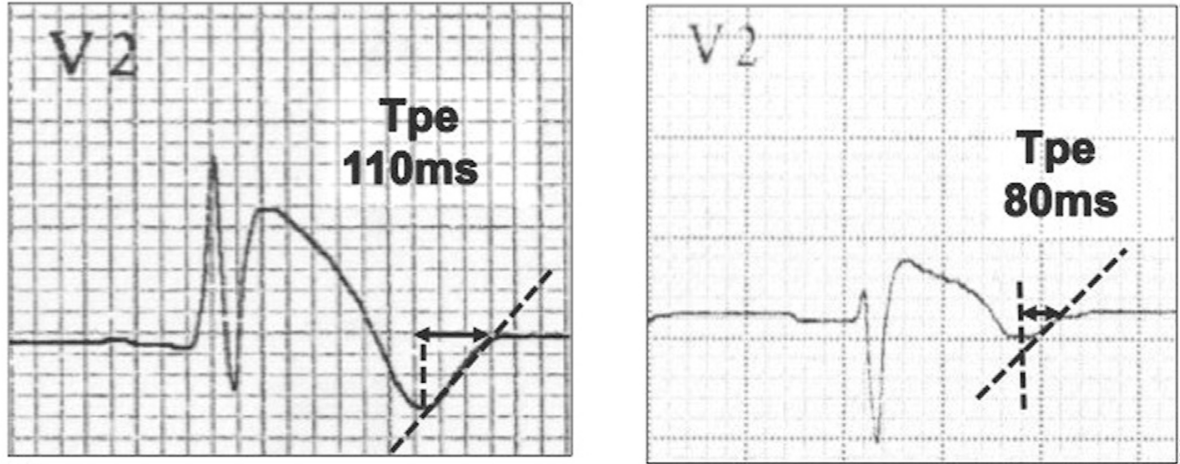

Figure 1 A: Methods for calculating the time interval between the peak and the end of the T wave (Tpe interval) in the presence of various types of ST elevation (left, coved ST-segment elevation with negative T wave; middle, saddle-back type ST elevation with positive T wave; right, no ST elevation and positive $\mathrm{T}$ waves). B: Examples of the Tpe interval in a patient who presented with sudden death (left) and in an asymptomatic patient (right). 
appropriate implantable cardioverter-defibrillator therapy (AT). The Tpe interval was correlated with the usual clinical and electrophysiological parameters and with the occurrence of syncope and malignant ventricular arrhythmias (SD and/ or AT).

\section{Testing of the results on a new group of patients with BS}

The Tpe interval was measured in ECG leads $V_{1}-V_{4}$ in 55 other symptomatic or asymptomatic patients with BS with type 1 ECG by 4 independents observers (P.M., A.R., J.B.G., and V.P.) in order to test the correlations on a new group of patients.

\section{Statistical analysis}

Continuous data are expressed as mean \pm standard deviation. Continuous variables were compared using nonparametric Mann-Whitney test, except in the case of normal distribution showing homoscedasticity in which an unpaired $t$ test was used. Categorical variables were compared using the $\chi^{2}$ test.

The intraclass correlation coefficient was used to evaluate the interobserver correlation. To define the discriminant power from the Tpe interval, Tpe/QT ratio, and max Tpe for the occurrence of arrhythmic events, we constructed receiver operating characteristic (ROC) curves. The curve point with a specificity of $>80 \%$ was labeled as the optimized cutoff point and used in the analysis of odds ratio (OR), sensitivity, and specificity.

Logistic regression was used to investigate the association between variables and arrhythmic events and to determine OR and $95 \%$ confidence interval (CI) for univariate and multivariate analysis. Multivariate analysis was performed using the variables significantly associated with univariate analysis (with $P<.1$ ), and the number of variables allowed to be included in the model was dictated by the number of arrhythmic events. Since a few events occurred during follow-up, and for a more simple presentation of the results, cardiac events both at the initial presentation and during follow-up were combined for statistical analysis. Analysis and calculations were performed using the StatView program (1992-1996, version 5.0, Abacus Concepts, Inc., Berkeley, CA,). A $P$ value of $<.05$ was considered statistically significant for each analysis.

\section{Results}

Three hundred twenty-five consecutive patients with BS were included. With a few exceptions, the studied population has already been described in our previous reports. ${ }^{16,17}$ Hundred seventeen patients were included in the FINGER (France, Italy, The Netherlands, and Germany) Brugada Syndrome Registry, ${ }^{2}$ with 36 of them and 30 additional patients being also included in a previous study. ${ }^{18}$ Most patients were index cases (only 35 related cases from 14 families). The characteristics of the patient population are listed in Table 1. An ICD had been implanted in 136 patients
(42\%): because of resuscitated SD in 10, unexplained syncope in 33, inducibility at electrophysiology study in 77 , family history of SD in 12, and physician's choice in 4 .

At the initial presentation, 235 patients were asymptomatic while 80 patients complained of unexplained syncope and 10 patients had been resuscitated from SD. During a mean follow-up of $48 \pm 34$ months, 2 and 7 of the initially asymptomatic patients experienced SD and presented with AT, respectively, while 0 and 7 patients initially presenting with unexplained syncope experienced SD and AT, respectively. Including cardiac events and symptoms at diagnosis and during follow-up, 226 patients (70\%) were asymptomatic and 99 patients $(30 \%)$ have presented with a cardiac event: unexplained syncope in $73(22 \%)$ and documented ventricular arrhythmias in $26(8 \%)(12$ with SD and 14 with AT).

Measurements of QT and QT peak intervals were feasible in $94 \%$ of the cases ( 74 measurements could not be performed mainly because of not discernible $\mathrm{T}$ wave). The intraclass correlation coefficients were between .71 and .82 for the Tpe measurement.

There was no significant difference in Tpe intervals, and except in lead $V_{1}$, there were only a few borderline differences in the Tpe/QT ratio between patients with baseline type $1 \mathrm{ST}$ elevation and patients in whom ST elevation was elicited by drugs (Table 2). The QT interval was larger in patients with a Tpe interval of $\geq 100 \mathrm{~ms}$, but there was no difference in QRS duration or QT peak values according to the presence of a Tpe interval $<100 \mathrm{~ms}$ or $\geq 100 \mathrm{~ms}$ (Table 3).

The corrected QT intervals in any lead were not significantly correlated with the occurrence of SD/AT or syncope. The Tpe interval and Tpe/QT ratio in leads $\mathrm{V}_{1}, \mathrm{~V}_{2}, \mathrm{~V}_{3}, \mathrm{~V}_{4}$ as well as max Tpe and Tpe dispersion were longer in patients with malignant ventricular arrhythmias (SD and/or AT) than in patients with syncope, who displayed longer values than did asymptomatic patients (Table 4). Differences were highly significant between patients with SD/AT and asymptomatic patients or between patients with syncope and asymptomatic patients, while differences between patients

Table 1 Characteristics of the patient population

\begin{tabular}{lc}
\hline Age $(\mathrm{y})$ & $47 \pm 13$ \\
\hline Sex: male & $259 / 325(80)$ \\
Spontaneous type 1 ST elevation & $143 / 325(44)$ \\
ST elevation after drug challenge & $182 / 325(56)$ \\
Fragmented QRS complex & $8 / 325(2.5)$ \\
Family history of SD & $94 / 325(29)$ \\
History of atrial fibrillation & $26 / 325(8)$ \\
Genetic testing & $190 / 325(58)$ \\
SCN5A gene mutations & $43 / 190(23)$ \\
ST elevation in the peripheral ECG leads & $30 / 325(9)$ \\
First-degree atrioventricular block & $114 / 325(35)$ \\
Electrophysiology study & $218 / 325(67)$ \\
Inducible at electrophysiology study & $92 / 218(42)$ \\
\hline
\end{tabular}

Values are presented as mean \pm standard deviation or as $\mathrm{n} / \mathrm{N}(\%)$. ECG = electrocardiographic; $S D=$ sudden death. 
Table 2 Tpe interval and Tpe/QT ratio according to the presence of spontaneous or drug-induced ST elevation

Class 1 drug-

Spontaneous induced

type 1 ECG type 1 ECG

Variable

$(n=143)$

$$
(n=182)
$$

Tpe interval in lead $V_{1}(\mathrm{~ms})$

Tpe $V_{2}$ (ms)

Tpe $V_{3}$ (ms)

Tpe $V_{4}$ (ms)

Tpe $V_{5}(\mathrm{~ms})$

Tpe $V_{6}$ (ms)

Max Tpe (ms)

Tpe dispersion

Tpe/QT ratio in lead $V_{1}$

Tpe/QT $V_{2}$

Tpe/QT $V_{3}$

Tpe/QT $V_{4}$

Tpe/QT $V_{5}$

Tpe/QT $V_{6}$

Heart rate (beats/min)

\begin{tabular}{lll}
$69 \pm 21$ & $65 \pm 19$ & .08 \\
$71 \pm 22$ & $73 \pm 24$ & .5 \\
$76 \pm 23$ & $76 \pm 23$ & .9 \\
$75 \pm 18$ & $73 \pm 22$ & .6 \\
$68 \pm 16$ & $70 \pm 17$ & .5 \\
$67 \pm 17$ & $67 \pm 19$ & .6 \\
$89 \pm 21$ & $86 \pm 22$ & .2 \\
$35 \pm 20$ & $34 \pm 20$ & .7 \\
$0.18 \pm 0.04$ & $0.17 \pm 0.04$ & .01 \\
$0.18 \pm 0.05$ & $0.18 \pm 0.06$ & .8 \\
$0.20 \pm 0,06$ & $0.19 \pm 0.06$ & .5 \\
$0.20 \pm 0,04$ & $0.19 \pm 0.05$ & .04 \\
$0.18 \pm 0,04$ & $0.18 \pm 0.05$ & .6 \\
$0.18 \pm 0.04$ & $0.17 \pm 0.05$ & .08 \\
$69 \pm 19$ & $68 \pm 20$ & .6 \\
\hline
\end{tabular}

Values are presented as mean \pm standard deviation.

$\mathrm{ECG}=$ electrocardiogram; Max Tpe = maximum value of the time interval between the peak and the end of the T wave; Tpe interval = time interval between the peak and the end of the $T$ wave.

with SD/AT and patients with syncope were nonsignificant (borderline value for max Tpe). Box plots for max Tpe according to the 3 groups of patients are shown in Figure 2 .

In order to test whether these results were linked to the presence of type 1 ST elevation and whether an increased Tpe interval may be simply due to a deeper negative $T$ wave (Figure 1B), we compared the presence of type 1 ST elevation in ECG leads V1-V4 to the distribution of max Tpe over the precordial leads. Type 1 ST elevation was mostly present in lead $V_{1}$ (292 of 325) and lead $V_{2}$ (235 of 325 ), more rarely in lead $V_{3}$ (24 of 325$)$, and exceptionally in lead $\mathrm{V}_{4}$ (4 of 325), while max Tpe was equally distributed from lead $\mathrm{V}_{1}$ to lead $\mathrm{V}_{4}$ (lead $\mathrm{V}_{1}$ : 103 of 325 ; lead $\mathrm{V}_{2}$ : 152 of 325 ; lead $V_{3}: 150$ of 325 ; lead $V_{4}: 139$ of 325 ), regardless of

Table 3 QRS duration, QT interval duration, and QT peak values according to the presence of a Tpe interval $<100 \mathrm{~ms}$ or $\geq 100 \mathrm{~ms}$

\begin{tabular}{|c|c|c|c|}
\hline Variable & $\begin{array}{l}\text { Tpe interval } \\
<100 \mathrm{~ms}\end{array}$ & $\begin{array}{l}\text { Tpe interval } \\
\geq 100 \mathrm{~ms}\end{array}$ & $P$ \\
\hline QRS duration (ms) & $108 \pm 19$ & $110 \pm 19$ & .4 \\
\hline QT interval in lead $V_{1}$ (ms) & $394 \pm 67$ & $445 \pm 75$ & .0001 \\
\hline QT peak $V_{1}(\mathrm{~ms})$ & $332 \pm 66$ & $339 \pm 74$ & .6 \\
\hline QT $V_{2}(\mathrm{~ms})$ & $416 \pm 77$ & $456 \pm 86$ & .0008 \\
\hline QT peak $V_{2}(\mathrm{~ms})$ & $352 \pm 76$ & $347 \pm 85$ & .6 \\
\hline QT V $\mathrm{V}_{3}(\mathrm{~ms})$ & $399 \pm 69$ & $440 \pm 78$ & $<.0001$ \\
\hline QT peak $V_{3}(\mathrm{~ms})$ & $332 \pm 70$ & $330 \pm 79$ & .8 \\
\hline QT $\mathrm{V}_{4}(\mathrm{~ms})$ & $398 \pm 69$ & $431 \pm 79$ & .004 \\
\hline QT peak $V_{4}(\mathrm{~ms})$ & $330 \pm 68$ & $323 \pm 78$ & .5 \\
\hline QT $V_{5}(\mathrm{~ms})$ & $389 \pm 66$ & $438 \pm 71$ & .001 \\
\hline QT peak $V_{5}(\mathrm{~ms})$ & $323 \pm 67$ & $332 \pm 67$ & .5 \\
\hline QT $\mathrm{V}_{6}(\mathrm{~ms})$ & $391 \pm 66$ & $461 \pm 107$ & $<.0001$ \\
\hline QT peak $V_{6}(\mathrm{~ms})$ & $326 \pm 66$ & $356 \pm 102$ & .06 \\
\hline
\end{tabular}

the spontaneous or drug-induced ST elevation and regardless of whether patients were symptomatic or not.

The area under ROC curves for the Tpe interval in leads $\mathrm{V}_{1}-\mathrm{V}_{4}$, Tpe dispersion, and max Tpe was 0.7 (for each lead $\mathrm{V}_{1}, \mathrm{~V}_{2}, \mathrm{~V}_{3}$, and $\left.\mathrm{V}_{4}\right), 0.62$, and 0.79 , respectively. No additional information was given by the ROC curves for Tpe/QT ratios. According to this observation, the best performing variable was max Tpe (Figure 3). An optimized cutoff point was determined from the ROC curve for this variable with respect to SD/AT: a max Tpe of $\geq 100 \mathrm{~ms}$ in any precordial lead has a sensitivity of $84 \%$, a specificity of $68 \%$, a positive predictive value of $19 \%$, and a negative predictive value of $98 \%$ for the occurrence of any ventricular arrhythmic event. A max Tpe of $\geq 100 \mathrm{~ms}$ was present in 47 of 226 asymptomatic patients $(21 \%)$, in 48 of 73 patients with syncope (66\%), and in 22 of 26 patients with SD/AT (85\%) (relative risk 3.4; $P<.0001$ ).

Correlations between max Tpe $\geq 100 \mathrm{~ms}$ and other clinical and electrophysiological variables are listed in Table 5. A max Tpe of $\geq 100 \mathrm{~ms}$ was significantly correlated with male sex, the presence of an ICD, the presence of fascicular block, and type 1 ST elevation in the peripheral leads.

Correlations between clinical and electrophysiological factors and the occurrence of any ventricular arrhythmic event (SD/AT) in univariate or multivariate analysis are listed in Table 6. A max Tpe of $\geq 100 \mathrm{~ms}$ was the most powerful parameter associated with outcome in multivariate analysis (OR 9.61; 95\% CI 3.13-29.41; $P<.0001$ ). This was also the case in the subgroups of patients with spontaneous ST elevation (OR 8.40; 95\% CI 2.21-32.26; $P=.002$ ) and patients without spontaneous ST elevation (OR 14.08; 95\% CI 1.65-125; $P=.01$ ).

This remained significant in the subgroup of ICD-implanted patients $(\mathrm{n}=136$; OR $6.09 ; 95 \%$ CI 1.9-19.6; $P=.002)$. In the nonimplanted patients $(\mathrm{n}=189)$, there was no significant association between max Tpe $\geq 100 \mathrm{~ms}$ and SD in logistic regression, but there was only 2 SDs in this population: both SDs occurred in patients with a max Tpe of $\geq 100 \mathrm{~ms}$ ( 2 of 51) vs none in other patients ( 0 of 138) $(P=.02)$.

For the subgroup of initially asymptomatic patients $(n=235)$, a max Tpe of $\geq 100$ ms remained associated with the occurrence of SD/AT (OR 13.3; 95\% CI 2.68-66.6; $P=.001$ ), but this was significant only for patients with spontaneous type 1 ST elevation (OR 15.87; 95\% CI 2.75-90.9; $P=.002$ ) (7 of these initially asymptomatic patients with spontaneous type 1 will present with AT, and 5 of them had a max Tpe of $\geq 100 \mathrm{~ms}$ ). In patients without baseline type $1 \mathrm{ECG}$, there was no significant association between max Tpe $\geq 100 \mathrm{~ms}$ and SD/AT in logistic regression, but there was only 2 SDs in this population: both SDs occurred in patients with a max Tpe of $\geq 100 \mathrm{~ms}$ ( 2 of 37) vs none in other patients $(0$ of 103) $(P=.02)$.

\section{Testing of the correlations on a new group of patients with BS}

Of the 55 patients with BS with type 1 ECG (18 SDs, 17 patients presenting with syncope, and 20 asymptomatic 
Table 5 Correlations between an increased Tpe interval (max Tpe $>100 \mathrm{~ms}$ ) and other clinical and electrophysiological variables

\begin{tabular}{lccc}
\hline & $\begin{array}{c}\text { Max Tpe interval } \geq 100 \mathrm{~ms} \\
\text { Variable }\end{array}$ & $\begin{array}{c}\text { Max Tpe interval }<100 \mathrm{~ms} \\
(\mathrm{n}=117[36 \%])\end{array}$ & $P$ \\
\hline Age at diagnosis (y) & $47 \pm 14$ & $46 \pm 13$ & .7 \\
Sex: male & $100 / 117(85)$ & $159 / 208(76)$ & .05 \\
SCN5A mutation & $19 / 74(26)$ & .4 \\
Spontaneous type 1 ST elevation & $53 / 117(45)$ & $24 / 116(21)$ & .7 \\
Inducibility at electrophysiology testing & $38 / 78(48)$ & $90 / 208(43)$ & .15 \\
Family history of sudden death & $41 / 117(35)$ & $54 / 140(39)$ & .07 \\
Atrial fibrillation & $13 / 117(11)$ & $53 / 207(25)$ & .12 \\
Implantable-cardioverter defibrillator ${ }^{*}$ & $66 / 117(56)$ & $13 / 208(21)$ & $<.0001$ \\
PR interval (ms) & $184 \pm 36$ & $70 / 208(34)$ & .27 \\
First-degree atrioventricular block (PR interval $>200 \mathrm{~ms})$ & $44 / 115(38)$ & $182 \pm 35$ & .4 \\
Right bundle branch block & $35 / 115(30)$ & $70 / 207(34)$ & .4 \\
Fascicular block & $27 / 115(23)$ & $54 / 208(26)$ & .007 \\
QRS duration (ms) & $110 \pm 19$ & $25 / 208(12)$ & .47 \\
HV interval (ms) & $52 \pm 10$ & $108 \pm 19$ & .78 \\
Fragmented QRS & $1 / 115(1)$ & $52 \pm 10$ & .10 \\
Max J-point elevation (mm) & $3.7 \pm 1.5$ & $7 / 208(3.3)$ & .23 \\
Type 1 ST elevation in peripheral leads & $22 / 114(19)$ & $3.5 \pm 1.4$ & .0001 \\
Heart rate (beats/min) & $66 \pm 22$ & $8 / 207(4)$ & .08 \\
\hline
\end{tabular}

Values are presented as mean \pm standard deviation or as $\mathrm{n} / \mathrm{N}(\%)$.

ICD = implantable cardioverter-defibrillator; Max Tpe = maximum value of the time interval between the peak and the end of the T wave; Tpe interval $=$ time interval between the peak and the end of the T wave.

*The higher rate of ICD implantation in the subgroup of patients with max Tpe $\geq 100 \mathrm{~ms}$ suggests that max Tpe is correlated with malignant ventricular arrhythmias and that ICD had often been implanted in symptomatic patients (presence of an ICD was significantly correlated with the existence of sudden death or syncope; $P<.05)$.

and subepicardial myocardial cells and has been proposed as an ECG marker of transmural dispersion of repolarization when measured in the precordial leads. ${ }^{3,5,19}$ In humans, differences in endocardial and epicardial recovery intervals have been shown to correspond to the Tpe intervals on the surface ECG. ${ }^{20}$ The Tpe interval may represent an interesting parameter for exploring the repolarization process in BS. An increased Tpe interval in the precordial leads may be the electrocardiographical representation of major transmural heterogeneity in the ventricular repolarization process according to one of the proposed mechanisms underlying ST elevation in $\mathrm{BS}^{21}$ and thus possibly correlating with the arrhythmogenic risk.

Alternatively, on the basis of the "depolarization theory," ST elevation has been proposed to be linked to the conduction delays observed in the right ventricular outflow tract (RVOT) in patients with BS. During the time period corresponding to the QRS end and onset of repolarization,

Table 6 Correlations between clinical and electrophysiological factors and the occurrence of any ventricular arrhythmic event in univariate or multivariate analysis*

\begin{tabular}{ll}
\hline Variable & OR $(95 \%$ CI $)$ \\
\hline Univariate analysis & \\
Max Tpe $\geq 100 \mathrm{~ms}$ & $11.76(3.95-35.71)$ \\
Type 1 ST elevation in peripheral leads & $6.89(2.74-17.24)$ \\
Syncope & $3.09(1.37-6.98)$ \\
Spontaneous type 1 ST elevation & $2.59(1.12-6.00)$ \\
SCN5A mutation & $2.98(1.04-8.55)$ \\
First-degree atrioventricular block (PR interval $\geq 200 \mathrm{~ms})$ & $2.29(1.02-5.13)$ \\
Familial sudden death & $2.25(1.00-5.08)$ \\
QRS duration & $1.02(1.00-1.04)$ \\
Fragmented QRS & .006 \\
Inducibility at electrophysiology study & $.02 .06(0.78-21.28)$ \\
Sex: male & $2.43(0.85-6.97)$ \\
Multivariate analysis & $2.04(0.59-7.03)$ \\
Max Tpe $\geq 100$ ms & .045 \\
Type 1 ST elevation in peripheral leads & .05 \\
\hline
\end{tabular}

Max Tpe = maximum value of the time interval between the peak and the end of the T wave; Tpe interval = time interval between the peak and the end of the T wave.

* Due to the low number of arrhythmic events, only the 2 variables showing the strongest association in univariate analysis were included in the multivariate model. 
the membrane potential in the right ventricle is more positive than in the RVOT, leading to an extracellular current flowing from the right ventricle to the RVOT and a positive deflection in the right precordial leads (ST elevation) and the reverse occurs thereafter - that is, more positive membrane potentials at the RVOT than at the right ventricleleading to a negative T wave. ${ }^{22}$ Clinical arguments supporting the depolarization theory come from the high prevalence of late potentials or conduction disturbances on ECG in $\mathrm{BS},{ }^{16}$ from the presence of delayed epicardial fragmented potentials at the RVOT whose ablation led to ECG normalization, ${ }^{23}$ or from the simultaneous fragmentation and delay in epicardial RVOT electrograms and appearance of type 1 ST elevation during ajmaline perfusion. ${ }^{24}$ The results of our study do not allow us to argue in favor of any mechanism, although changes in terminal repolarization may be involved (see Table 3).

An increased Tpe interval has been shown to identify patients at higher risk of malignant arrhythmic events in patients with or without structural heart diseases. ${ }^{4-10}$ A few studies have also addressed this issue in BS. Castro Hevia et $\mathrm{al}^{11}$ were the first to link increased Tpe interval as a risk factor in patients with BS: in 29 patients, max Tpe in the precordial leads was significantly prolonged in patients with BS with recurrences versus patients with BS without events or controls, with a best discriminant max Tpe cutoff value of $\geq 100 \mathrm{~ms}$. The Tpe interval was found to be longer in symptomatic and/or inducible patients with BS vs patients with BS without events (who did not differ from controls) in a short series of 27 patients, with a Tpe interval of $\geq 120 \mathrm{~ms}$ being associated with higher cardiac events in patients with BS. ${ }^{12}$ In another series of 23 patients with BS, inducible subjects displayed an increased Tpe interval in lead $\mathrm{V}_{2}$ but no correlation with spontaneous events was made. ${ }^{13}$ The largest series to date included 200 patients with BS, but failed to find any significant difference in the Tpe interval in leads II and $\mathrm{V}_{2}$ between symptomatic and asymptomatic patients with BS. ${ }^{14}$ Calculation of the Tpe interval in the 6 precordial leads and by 2 independent observers blinded to the symptoms may explain the diverging results of this study ${ }^{14}$ and of the present study.

Some of these studies found increased Tpe interval to be associated with a poor prognosis but included only a limited number of patients, while other larger cohort studies failed in finding any prognosis role of the Tpe interval. Thus, our study is the first to demonstrate that an increased Tpe interval is highly and independently related to arrhythmic events in a large series of unselected patients with BS.

Risk stratification in BS remains an unsolved issue. Even if a lot of more or less accepted criteria have been proposed for selecting asymptomatic patients with BS prone to a significant risk of malignant ventricular arrhythmias and thus for suggesting primary ICD implantation, to date ICD in asymptomatic patients may only be considered in the case of spontaneous type 1 ECG and with inducible VF. ${ }^{1}$ In our series, we found that an increased Tpe interval had a clearly higher correlation with malignant ventricular arrhythmias (OR $\sim 10)$ than did the usual parameters used in risk stratification. Large prospective trials are needed to confirm these results.

We speculated that an increased Tpe interval could only be the result of a larger or deeper negative $T$ wave in the right precordial leads - the more deeper the $\mathrm{T}$ wave, the more longer the Tpe interval-which may have render ECG analysis easier. Moreover, deeper $\mathrm{T}$ waves would implicitly have the same meaning as increased Tpe interval in terms of electrophysiological mechanism underlying BS and higher arrhythmogenic risk. However, max Tpe was equally present in leads $V_{1}$ to $V_{4}$ while type $1 \mathrm{ST}$ elevation was rare in lead $\mathrm{V}_{3}$ and exceptional in lead $\mathrm{V}_{4}$. Thus, the correlation between max Tpe with the presence of type 1 ECG or with leads exploring the RVOT, and an increased Tpe interval may be the manifestation of more subtle changes in the repolarization process in BS than the crude type 1 ECG pattern observed in the right precordial leads.

\section{Study limitations}

This was a retrospective study of a large series of patients with BS, and the value of an increased Tpe interval for risk stratification in BS should ideally now be confirmed in prospective trials.

Although interobserver variability was shown to be acceptable, measurements of the Tpe interval remain difficult and probably sometimes unreliable especially at a paper speed of $25 \mathrm{~mm}$ on the ECG. Achieving better reliability using a higher paper speed or automated ECG interpretation deserves further studies. However, our findings were confirmed by 4 independent Tpe interval measurements in an additional group of patients with BS.

A max Tpe of $\geq 100 \mathrm{~ms}$ was independently correlated with arrhythmic events in the subgroup of ICD-implanted patients only. This is probably due to the recording by the device of nonsustained ventricular arrhythmias destined to terminate spontaneously and those would have remained undetected/ untreated in the absence of an ICD. However, this means that an increased Tpe interval is clearly related to arrhythmogenesis in patients with BS. The low number of SDs in nonimplanted patients possibly explains the lack of significant correlation between max Tpe $\geq 100 \mathrm{~ms}$ and symptomatic ventricular arrhythmias in logistic regression: actually, malignant ventricular arrhythmias in this subgroup occurred only in patients with a max Tpe of $\geq 100 \mathrm{~ms}(P<.05)$. The same occurred in asymptomatic patients without spontaneous type 1 ECG (not correlated with arrhythmic events in regression analysis, but the only 2 patients with SD/AT in this subgroup had a max Tpe of $\geq 100 \mathrm{~ms} ; P<.05$ ). Hence, an increased Tpe interval remained significantly associated with the occurrence of ventricular arrhythmia regardless of whether the patients were implanted with an ICD or not and regardless of whether type 1 ST elevation in asymptomatic patients was present at baseline or not.

\section{Conclusion}

Risk stratification in BS remains difficult and controversial. The transmural dispersion of repolarization may be 
approached by the Tpe interval. A max Tpe of $\geq 100 \mathrm{~ms}$ in the precordial leads was highly related to malignant ventricular arrhythmias in this large series of patients with BS. Once prospectively evaluated, this simple ECG parameter could be proposed for refining risk stratification in BS in addition to accepted or other recently recognized markers.

\section{References}

1. Priori SG, Wilde AA, Horie M, et al. HRS/EHRA/APHRS expert consensus statement on the diagnosis and management of patients with inherited primary arrhythmia syndromes. Heart Rhythm 2013;10:1932-1963.

2. Probst V, Veltmann C, Eckardt L, et al. Long-term prognosis of patients diagnosed with Brugada syndrome: results from the FINGER Brugada Syndrome Registry. Circulation 2010;121:635-643.

3. Antzelevitch C, Yan GX, Shimizu W. Transmural dispersion of repolarization and arrhythmogenicity: the Brugada syndrome versus the long QT syndrome. J Electrocardiol 1999;32:158-165.

4. Gupta P, Patel C, Patel H, Narayanaswamy S, Malhotra B, Green JT, Yan GXT. (p-e)/QT ratio as an index of arrhythmogenesis. J Electrocardiol 2008;41: $567-574$.

5. Antzelevitch C, Sicouri S, Di Diego JM, Burashnikov A, Viskin S, Shimizu W, Yan GX, Kowey P, Zhang L. Does Tpeak-Tend provide an index of transmural dispersion of repolarization? Heart Rhythm 2007;4:1114-1116.

6. Tatlisu MA, Özcan KS, Güngör B, Ekmekçi A, Çekirdekçi EI, Aruğarslan E, Çinar T, Zengin A, Karaca M, Eren M, Erdinler I. Can the T-peak to T-end interval be a predictor of mortality in patients with ST-elevation myocardial infarction? Coron Artery Dis 2014;5:399-404.

7. Karim Talib A, Sato N, Sakamoto N, Tanabe Y, Takeuchi T, Saijo Y, Kawamura Y, Hasebe N. Enhanced transmural dispersion of repolarization in patients with J wave syndromes. J Cardiovasc Electrophysiol 2012;23:1109-1114.

8. Smetana P, Schmidt A, Zabel M, Hnatkova K, Franz M, Huber K, Malik M. Assessment of repolarization heterogeneity for prediction of mortality in cardiovascular disease: peak to the end of the $\mathrm{T}$ wave interval and nondipolar repolarization components. J Electrocardiol 2011;44:301-308.

9. Anttonen $\mathrm{O}$, Väänänen $\mathrm{H}$, Junttila J, Huikuri HV, Viitasalo M. Electrocardiographic transmural dispersion of repolarization in patients with inherited short QT syndrome. Ann Noninvasive Electrocardiol 2008;13:295-300.

10. Aiba T, Shimizu W, Inagaki M, Satomi K, Taguchi A, Kurita T, Suyama K, Aihara N, Sunagawa K, Kamakura S. Excessive increase in QT interval and dispersion of repolarization predict recurrent ventricular tachyarrhythmia after amiodarone. Pacing Clin Electrophysiol 2004;27:901-909.

11. Castro Hevia J, Antzelevitch C, Tornés Bárzaga F, Dorantes Sánchez M, Dorticós Balea F, Zayas Molina R, Quiñones Pérez MA, Fayad Rodríguez Y. Tpeak-Tend and Tpeak-Tend dispersion as risk factors for ventricular tachycardia/ventricular fibrillation in patients with the Brugada syndrome. J Am Coll of Cardiol 2006;47: 1828-1834.

12. Wang JF, Shan QJ, Yang B, Chen ML, Zou JG, Chen C, Xu DJ, Cao KJ. TpeakTend interval and risk of cardiac events in patients with Brugada syndrome. Zhonghua Xin Xue Guan Bing Za Zhi 2007;35:629-632.

13. Letsas KP, Weber R, Astheimer K, Kalusche D, Arentz T. Tpeak-Tend interval and Tpeak-Tend/QT ratio as markers of ventricular tachycardia inducibility in subjects with Brugada ECG phenotype. Europace 2010;12:271-274.

14. Junttila MJ, Brugada P, Hong K, et al. Differences in 12-lead electrocardiogram between symptomatic and asymptomatic Brugada syndrome patients. J Cardiovasc Electrophysiol 2008; 19:380-383.

15. Rautaharju PM, Surawicz B, Gettes LS, et al. AHA/ACCF/HRS recommendations for the standardization and interpretation of the electrocardiogram, Part IV: the ST segment, T and U waves, and the QT interval. Circulation 2009;119: e241-e250.

16. Maury P, Rollin A, Sacher F, et al. Prevalence and prognostic role of various conduction disturbances in patients with the Brugada syndrome. Am J Cardiol 2013;112:1384-1389.

17. Rollin A, Sacher F, Gourraud JB, et al. Prevalence, characteristics, and prognosis role of type 1 ST elevation in the peripheral ECG leads in patients with Brugada syndrome. Heart Rhythm 2013;10:1012-1018.

18. Sacher F, Probst V, Maury P, et al. Outcome after implantation of a cardioverterdefibrillator in patients with Brugada syndrome: a multicenter study, Part 2. Circulation 2013;128:1739-1747.

19. Yan GX, Antzelevitch C. Cellular basis for the normal $T$ wave and the electrocardiographic manifestations of the long-QT syndrome. Circulation 1998;98:1928-1936.

20. Chinushi M, Sato A, Izumi D, Furushima H. Nifekalant enlarged the transmural activation-recovery interval difference as well as the peak-to-end interval on surface ECG in a patient with short-QT syndrome. J Cardiovasc Electrophysiol 2012;23:877-880.

21. Yan GX, Antzelevitch C. Cellular basis for the Brugada syndrome and other mechanisms of arrhythmogenesis associated with ST-segment elevation. Circulation 1999;100:1660-1666.

22. Meregalli PG, Wilde AA, Tan HL. Pathophysiological mechanisms of Brugada syndrome: depolarization disorder, repolarization disorder, or more? Cardiovasc Res 2005;67:367-378.

23. Nademanee K, Veerakul G, Chandanamattha P, Chaothawee L, Ariyachaipanich A, Jirasirirojanakorn K, Likittanasombat K, Bhuripanyo K, Ngarmukos T. Prevention of ventricular fibrillation episodes in Brugada syndrome by catheter ablation over the anterior right ventricular outflow tract epicardium. Circulation 2011;29(123): $1270-1279$.

24. Sacher F, Jesel L, Jais P, Haïssaguerre M. Insight into the mechanism of Brugada syndrome: epicardial substrate and modification during ajmaline testing. Heart Rhythm 2014;11:732-734. 